\title{
Neonatal Ileo-Ileal and Ileo-Cloacal Exstrophic Synchronous Dual Intussusceptions in a Preterm Infant With Cloacal Exstrophy
}

\author{
Ramnik Patel ${ }^{\mathrm{a}}$, Yew W. Tan ${ }^{\mathrm{a}}$, Sumant Patil ${ }^{\mathrm{a}}$, Abraham Cherian ${ }^{\mathrm{b}}$, Bharat More ${ }^{\mathrm{a}, \mathrm{c}}$
}

\begin{abstract}
We report an ex-35 weeks gestational age preterm female infant with prenatally diagnosed cloacal exstrophy who developed ileo-ileal and ileo-exstrophic dual synchronous intussusceptions at the corrected age of 3 weeks. The infant presented with acute illness. Initial fluid resuscitation and antibiotic treatment improved clinical condition but developed bleeding and increasingly discolouration of the distal loop associated with metabolic acidosis and concern for bowel obstruction and ischemia. Radiographs were non-specific. At exploration an irreducible necrotic advanced ileo-ileal intussusception and a reducible but viable ileo-exstrophic intussusception were found. Resection of necrotic ileo-ileal intussusception with ileostomy formation on the proximal end and closure of the distal ileum with reduction of ileoexstrophic intussusception was carried out uneventfully with good prognosis.
\end{abstract}

Keywords: Cloacal exstrophy; Ileo-ileal; Dual intussusception; Ileoexstrophic; Neonatal; Synchronous

\section{Introduction}

Neonatal intussusception is very rare in term babies and exceptional in the premature infants [1-8]. Acute neonatal intestinal obstruction generally happens at one level. Usually proximal obstruction leads to collapse of the distal bowel and second strangulating obstruction downstream at the same time is an unexpected event. Intussusception is an acute strangulating generally a solitary lesion and synchronous dual intussuscep-

\footnotetext{
Manuscript accepted for publication March 17, 2016

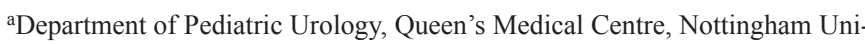
versity Hospitals NHS Foundation Trust, Derby Road, Nottingham NG7 2UH, UK

bDepartment of Pediatric Urology, Great Ormond Street Hospital NHS Foundation Trust and University College Hospital, London, UK

${ }^{\mathrm{c} C}$ Corresponding Author: Bharat More, Department of Pediatric Urology, Queen's Medical Centre, Nottingham University Hospitals NHS Foundation Trust, Derby Road, Nottingham NG7 2UH, UK. Email: bharat.more@nhs.net
}

doi: http://dx.doi.org/10.14740/wjnu253w tions have not been reported earlier.

\section{Case Report}

A preterm female infant with corrected age of 3 weeks, a case of prenatally diagnosed and postnatally confirmed cloacal exstrophy awaiting surgery that had recent respiratory syncytial virus (RSV) positive bronchiolitis became acutely unwell with suspected sepsis.

Infant was born at 35 weeks with birth weight of 2,200 g by cesarean section. The infant had left ectopic pelvic kidney, spina bifida occulta with lipo-myelo-meningocele and partial sacral agenesis.

The infant presented with acute collapse at a district gen-

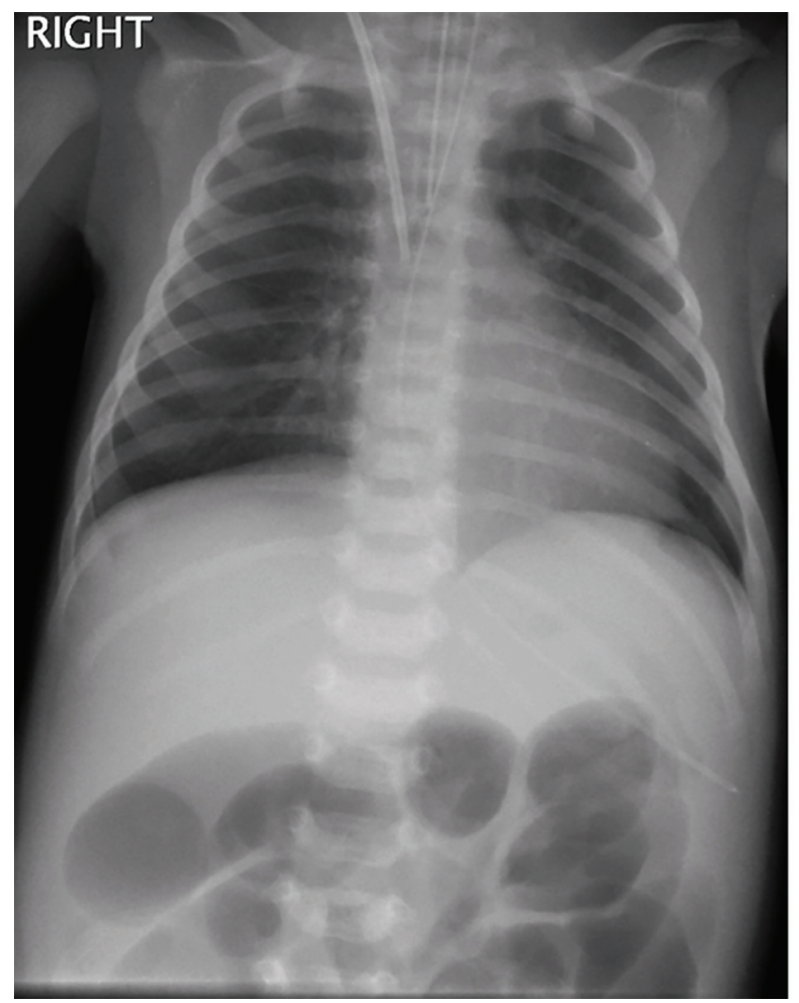

Figure 1. Chest radiograph showing dilated bowel loops in upper abdomen. 


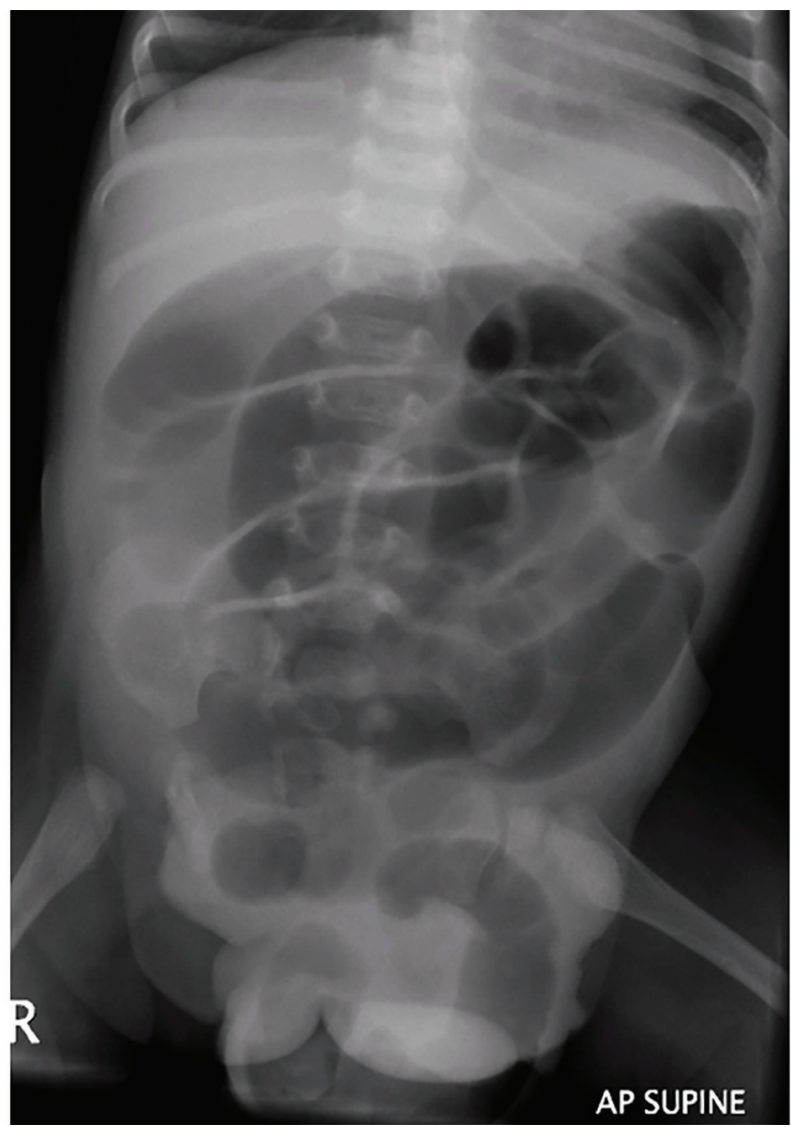

Figure 2. Abdominal radiograph demonstrated decompressed stomach, dilated bowel with vene conniventes and characterless loops and prolapsed loop through cloacal exstrophy. Note partial sacral dysgenesis, spina bifida and widening of the pubic symphysis in the background.

eral hospital associated with persistent crying, irritability, vomiting, absolute constipation, abdominal distension, collapse, dehydration, features of sepsis and concern about necrotic prolapsed bowel at the cloacal exstrophy. She weighed 2,570 g. The peripheries were cold and clammy. Necrotizing enterocolitis (NEC) was suspected. Initial fluid resuscitation $40 \mathrm{~mL} / \mathrm{kg}$ and triple antibiotic treatment (cefotaxime, gentamycin and metronidazole) improved clinical condition.

However, on the following day, she developed bleeding and increasingly dark purple discolouration with oozing of blood from the surface mucosa of the prolapsed distal elephant trunk like loop at the cloacal exstrophy associated with metabolic acidosis and concern regarding necrotic bowel.

The infant was pale, lethargic, apyrexial, tachycardic, tachypneic with distended tender abdomen. The discoloured, dry and dusky prolapsed elephant trunk like bowel at the cloacal exstrophy with no bowel contents through the lumen was seen. There was very little urine from the open ureteric orifices.

The capillary blood gases showed $\mathrm{pH}$ 7.1, $\mathrm{PCO}_{2}$ 5.1, $\mathrm{PO}_{2}$ 5.8 , bicarbonate $11 \mathrm{mmol} / \mathrm{L}$, lactate $2.4 \mathrm{mmol} / \mathrm{L}$, and base excess -16 . Complete blood count showed hemoglobin drop from $113 \mathrm{~g} / \mathrm{L}$ on the day of admission to $106 \mathrm{~g} / \mathrm{L}$ on the fol-
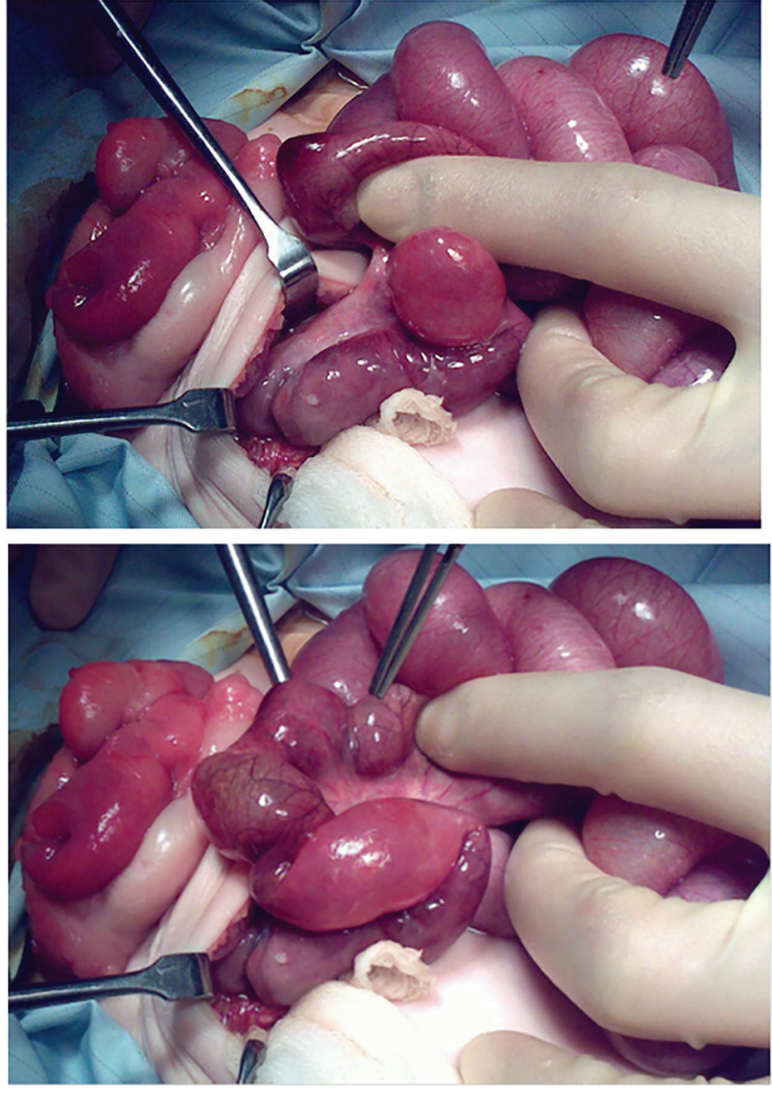

Figure 3. Findings and operative procedures. Note the ileo-ileal intussusception and prolapsed discoloured ileo-exstrophy.

lowing day, white cell count $13.13 \times 10^{9} / \mathrm{L}$, neutrophils 12.19 $\times 10^{9} / \mathrm{L}$ and platelets $487 \times 10^{9} / \mathrm{L}$. The renal function showed sodium $111 \mathrm{mmol} / \mathrm{L}$, potassium $6.11 \mathrm{mmol} / \mathrm{L}$, chloride 81 $\mathrm{mmol} / \mathrm{L}$, urea 41.2 , creatinine 66 , and C-reactive protein $<$ $2 \mathrm{mg} / \mathrm{L}$.

Chest radiograph showed clear lung fields (Fig. 1). Abdominal radiograph showed dilated bowel loops (Fig. 2). There were no signs of sepsis on chest radiograph and NEC on abdominal radiograph. As she continued to deteriorate, she was referred to and transferred to us on the third day of admission for small bowel obstruction with bowel ischemia.

The infant underwent exploratory laparotomy at which a necrotic proximal ileo-ileal intussusception in association with viable reducible distal prolapsed ileo-exstrophic intussusception with dusky but viable bowel was found. The incidental finding included duodenojejunal junction in the midline with few bands across it which were divided. Resection of proximal ileo-ileal necrotic intussusception and ileostomy formation of the proximal ileum, closure of the distal ileal stump and reduction of ileo-exstrophic intussusception concluded operation (Fig. 3).

The post-operative period was uneventful and she made uneventful recovery. The infant subsequently underwent successful closure of the exstrophy with bilateral pelvic osteotomies and currently is awaiting bladder neck reconstruction. 


\section{Discussion}

Peak age of intussusception is 5 - 9 months. Neonatal intussusception in a preterm infant is very rare, and is usually solitary and ileo-ileal in nature. Multiple sequential intussusceptions in a preterm infant have been reported but none of the synchronous proximal and distal intussusceptions in a preterm neonate [7]. A recent case and only case of ileo-colic intussusception in a preterm neonate has been described but none of the ileoexstrophic intussusception [8].

Our patient had recent RSV positive bronchiolitis and she was treated for suspected sepsis. The NEC is common but the clinical, laboratory and radiological features were not correlated well. The dilated bowel loops were considered secondary to septic ileus. However, our case is a reminder of the fact that index of suspicion for intussusception should be high and the threshold for ultrasound scan of the abdomen should be low as typical features are absent and clinical and radiological assessment is difficult. The synchronous intussusceptions could explain the absence of red currant jelly due to dual levels of obstruction, the proximal being absolute and strangulating.

Most intussusceptions in term neonates are usually ileocolic while most previously reported preterm intussusceptions were jejunal or ileal in location [2-4, 6-8]. Term infants have some lead point while the preterm infants with intussusception do not have a lead point [6]. Multiple sequential intussusceptions may be due to perinatal hypoxia, hypovolemia, and dysmotility and post-NEC stricture formation may act as lead point in neonates [7].

The clinical symptoms and signs are very non-specific, radiological features are difficult to interpret and even ultrasound may not be able to detect due to excessive bowel gas as seen in a recent case [8]. In our case, the features of small bowel obstruction with possible strangulation due to delay in diagnosis were evident and malrotation with volvulus was the provisional diagnosis and dual synchronous intussusceptions was a pleasant exploratory surprise.

Recent RSV positive bronchiolitis and features of septic shock initially misled due to unusual group of symptoms and lack of red currant jelly did not allow one to suspect intussusception due to synchronous lesions. As the diagnosis is delayed, the associated morbidity and mortality is high and one should keep a high index of suspicion for an intussusception in such cases.
A rare and very interesting case of neonatal ileo-ileal and ileo-cloacal exstrophic dual synchronous intussusceptions presenting as acute septic illness initially leading to delay in diagnosis and successful outcome following resection and stoma formation for the former and the reduction of the distal intussusception led to smooth recovery. The infant subsequently underwent successful closure of the exstrophy with bilateral pelvic osteotomies. She is currently awaiting bladder neck reconstruction.

\section{Conflict of Interest}

None.

\section{References}

1. Rachelson MH, Jernigan JP, Jackson WF. Intussusception in the newborn infant with spontaneous expulsion of the intussusceptum; a case report and review of the literature. J Pediatr. 1955;47(1):87-94.

2. Wang NL, Yeh ML, Chang PY, Sheu JC, Chen CC, Lee HC, Hung HY, et al. Prenatal and neonatal intussusception. Pediatr Surg Int. 1998;13(4):232-236.

3. Avansino JR, Bjerke S, Hendrickson M, Stelzner M, Sawin R. Clinical features and treatment outcome of intussusception in premature neonates. J Pediatr Surg. 2003;38(12):1818-1821.

4. Martinez BM, Gracia Alix A, Luisa del HM, et al. Intussusception in preterm neonate: a very rare major intestinal problem-systemic review of cases. J Perinat Med. 2004;32:190-194.

5. Anand PV, Ragupathy R, Kumaran V, et al. Neonatal intussusception: a surgical rarity. J Indian Assoc Pediatr Surg. 2004;9:198-200.

6. Ueki I, Nakashima E, Kumagai M, Tananari Y, Kimura A, Fukuda S, Hashimoto T. Intussusception in neonates: analysis of 14 Japanese patients. J Paediatr Child Health. 2004;40(7):388-391.

7. Slam KD, Teitelbaum DH. Multiple sequential intussusceptions causing bowel obstruction in a preterm neonate. J Pediatr Surg. 2007;42(7):1279-1281.

8. Shad J, Biswas R. Ileo-colic intussusception in premature neonate. BMJ Case Rep. 2011;2011. 